\title{
Announcement Arthritis Research: the move to publish research articles in full online only
}

When Arthritis Research was launched in 1999 the publishers and ourselves took the innovative decision to make all primary research articles available to everyone for free through the journal's own website (http://arthritisresearch.com) as well as through PubMed Central (http://www.pubmedcentral.nih.gov/), the National Institute of Health's repository for biomedical research articles, and through the BioMed Central (BMC) website (http://biomedcentral.com). We now feel that it is time to lead the way forward again by only publishing research articles in full online beginning with volume 4 number 4 of Arthritis Research.

Because research articles in Arthritis Research have always been free access, and because the main medium of publication of the journal has always been the Internet (the website provides the complete contents of the journal while the print edition contains selected items), very many more people already see the articles on the web than in print. Few, we expect, will mourn the fact that only the abstract (and a key illustration) of each paper will appear in print. We believe that online publishing of research articles will become the norm and see this as an ideal opportunity to develop Arthritis Research even further.

\section{Advantages to authors}

Publishing research articles in full only online has many advantages for Arthritis Research authors:

- The current length restrictions, which are tied to the print journal, can be removed allowing all the necessary data to be published as an integral part of the article, instead of as supplementary material that is already only available online.

- $\quad$ Papers can be published with their full and final citation immediately with no delay or loss of priority, which happens when a paper has to wait until it is printed for its citation.

- Articles, with their full citation, can be sent at an earlier stage to PubMed, meaning that they will be noticed more quickly.

- Colour figures can be included without concern for the costs to the author of reproducing them in print.

\section{How will it work?}

Each research article will be published online as soon as it is ready. The abstract of each article, along with a key figure or table, will be printed in the next issue.

\section{Citation system}

Research articles will continue to have a citation that is similar to the current system. The only change is that there will be an 'article number' as well as a page range. For example, the first research article to be published in this way will have the citation Arthritis Res 2002, 4:R1.1-R1.6 (e.g. for a six-page article) and the second to be published will be Arthritis Res 2002, 4:R2.1-R2.9 (e.g. for a ninepage article).

\section{Your questions answered}

The move to publish research articles in full online only will not affect the permanence of their availability, which is assured by their deposition in PubMed Central (and in other repositories in future).

The ISI have assured us that the journal's Impact Factor, which will be published in Journal Citation Reports (http://www.isinet.com/isi/products/citation/jcr/) in 2003, will not be affected by having research articles in full online only.

Research papers are available online in both a web and a journal/PDF format with permanent free access, so they can be printed locally and distributed as required at no cost. If a higher quality printed version of an article is preferred there will still be the option to purchase reprints.

This move will take effect immediately and beginning with volume 4 number 4 research articles will be published in full online only, with the abstract appearing in the print journal. The new instructions for authors are available on the website (http://arthritis-research.com/info/instructions/primaryinstructions.asp) and in the print version of volume 4 number 3 . Any questions not answered here or in the instructions can be sent directly to the editorial office (editorial@arthritis-research.com).

Peter E Lipsky and Ravinder N Maini Editors-in-Chief 